\title{
ANALYSIS OF THE EFFECTS OF SUPERPLASTICIZER ADDITION AND WATER REDUCTION IN CONCRETE MIXTURE ON CONCRETE COMPRESSIVE STRENGTH
}

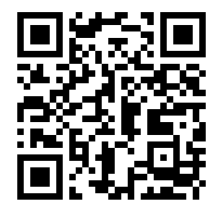

\author{
Dwi Sri Wiyanti ${ }^{*}{ }^{\otimes}$, Taufik Dwi Laksono ${ }^{2}$ \\ ${ }^{* 1,2}$ Civil Engineering, Faculty of Engineering, Wijayakusuma University Purwokerto, Jl. Beji \\ Kampus Karangsalam Purwokerto Indonesia 53152
}

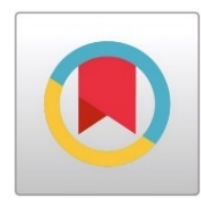

DOI: https://doi.org/10.29121/ijetmr.v7.i6.2020.688

Article Citation: Dwi Sri Wiyanti, and Taufik Dwi Laksono. (2020). ANALYSIS OF THE EFFECTS OF SUPERPLASTICIZER ADDITION AND WATER REDUCTION IN CONCRETE MIXTURE ON CONCRETE COMPRESSIVE STRENGTH. International Journal of Engineering Technologies and Management Research, 7(6), 47-57. https://doi.org/10.29121/ijetmr.v7 i6.2020.688

Published Date: 15 June 2020

Keywords:

Concrete

Contrete Compressive Strength

Cement

Superplasticizer

\begin{abstract}
Nowadays, concrete is still one of the most used building materials, it had been improved whether in it's quality or it's mixture materials. There were a lot of studies that had been conducted to improve concrete that related to the produced compressive strength, stiffening speed, flexibility, etc. There are so many materials that can be used in concrete mixture to improve compressive strength. This study was conducted by using superplasticizer and reducing water amount in concrete mixture and aimed to find out how big it's effects on the produced concrete compressive strength. Superplasticizer was used as admixture material wtih 1 percentage calculated by cement weight. Whereas water amount in concrete mixture was reduced by $0 \%, 15 \%$, and $17,5 \%$. The result obtained was that using 1\% superplasticizer and reducing $15 \%$ water amount in concrete mixture produced optimum compressive strength of 22,98 Mpa.
\end{abstract}

\section{INTRODUCTION}

The level of infrastructure needs that getting higher must be followed by the capabilty to meet the infrastructure with those needs. Innovations are continuously encouraged to provide maximum results for all parties so that the community's need for infrastructure can be fulfilled. Infrastructure construction nowadays whether by the government, the private sectors, or both of them have a positive impact on the development of construction industries.

The use of main materials in infrastructure construction holds an important role, one of those main materials is concrete. Concrete is still a part of the main materials because it has many advantages such as easy to shape, has the ability to withstand the load that works on it, and others.

Concrete can be use as a structural component such as stairs, beam, foundation, and another structural component (Wulfam, 2006:2), concrete can also be use as an architectural component like insulating wall. Day by day innovation on concrete is developing, for example the use of no-fine concrete where concrete does not includes sand in it's mixture (Diarto, 2014:1). Innovation on concrete is not only to improves it's quality but also to speed up the time and reducing the cost needed to acquire the desired concrete.

(C) 2020 The Author(s). This is an open access article distributed under the terms of the Creative Commons Attribution License, which permits unrestricted use, distribution, and reproduction in any medium, provided the original author and source are credited. 
Analysis of The Effects of Superplasticizer Addition and Water Reduction in Concrete Mixture on Concrete Compressive Strength

There are many studies on concrete especially the composition of concrete mixture materials. Rahmat, Irna and Syaiful (2016:218) in their study conclude that concrete with liquid addition material by 0,$25 ; 0,4 \%$ and $0,6 \%$ on the 7 th day and 14th day produced greater concrete compressive strength than normal concrete. Whereas Asrullah (2019:10) concludes that concrete compressive strength with sika concrete re-faired mortar addition by 5\% produced $311,89 \mathrm{~kg} / \mathrm{cm} 2$ greater concrete compressive strength than normal concrete. Syafruddin and Hastoro (2005:72) in their study that used superplasticizer the result is that reducing water by $30 \%$ and adding superplasticizer by 1,83\% able to produce average optimum compressive strength by 54 Mpa for 30 Mpa planned compressive strength. Santi Wahyuni Megasari and Winayati $2017: 117)$ state that average compressive strength is improving when adding Sikament NN by_1,3\% and 1.8\%.

Based on the above studies that have been conducted, whether material addition or material substitution in concrete mixture can improve concrete compressive strength. This study using superplasticizer addition with 1 percentage calculated by cement weight and water amount reduction in concrete's mixture to find out how big it's effect on the concrete compressive strength.

Concrete is a material that has strong compressive strength but weak tensile strength. Concrete can still be added with admixture material to change it's characteristics whether in fresh concrete form or hard concrete form. As a building structure, concrete is combined with steel truss to gain high performance whether as reinforced concrete or pre-stressed concrete (Paul and Antono, 2004:2).

According to Ali Asrono (2010:2), concrete is simply formed from the hardened mixture of cement, water, sand (fine aggregate), and crushed stone or gravel (coarse aggregate). Sometimes, to improve concrete quality, an admixture is added.

Superplasticizer (a high range water reducer admixture) is very good to improve mixture slick. Nowadays, superplasticizer has been developed to be able to be used on high-quality concrete and self-compact concrete. Superplasticizer has high flowability but self-compact concrete does not show segregation between aggregate and mortar so that able to reach every mold corner.

Some of the uses of a superplasticizer are facilitate the making of highly liquid concrete, reducing the need for water (25-35\%), and increasing workability, make it greater than ordinary water reducer (Paul and Antono, 2004:93).

According to Venu Malagavelli, Neelakanteswara Rao Paturu (2012:7), the use of different superplasticizer will produce significant improvement in the strength and workability of modified concrete.

Ali Hussein Hameed (2012:70), stated that the dosage of superplasticizer increase, the slump flow increases. This is expected because as the superplasticizer dosage increases the fluidity of the concrete also increases.

M. Benaicha, A. Hafidi Alaoui, O. Jalbaud, Y. Burtschell (2019:2068), stated that the compressive strength decreases with the increase of the superplasticizer dosage. In addition, the value of Slump flow diameter and $\mathrm{Hf} / \mathrm{Hi}$ ratio increase with the increase of the superplasticizer. On the other, the increase of superplasticizer decreases the $\mathrm{V}$-funnel flow time, the yield stress, and the plastic viscosity values.

\section{MATERIALS AND METHODS}

\subsection{CEMENT}

The cement that had used in this study was a cement that produced by PT. Sinar Tambang Artha Lestari namely Bima Cement. The cement functioned as coarse aggregate and fine aggregate binder material.

\subsection{FINE AGGREGATE}

The fine aggregate that had used was collected from Serayu River. Serayu River Sand that will be used as concrete mixture material must pass the grading test because that grading test result will be used in concrete mix design production process. 


\subsection{COARSE AGGREGATE}

Dwi Sri Wiyanti, and Taufik Dwi Laksono

The coarse aggregate that had used was crushed stones from stone crusher. The coarse aggregate was also grading tested to produce the concrete mix design.

\subsection{WATER}

Water is concrete mixture material that causes a reaction with cement, the water that used must not contain oil, acid, alkali, organic materials, and any other materials that can damage concrete. In this case, a drinkable clean water was used.

\subsection{THE SUPERPLASTICIZER THAT WAS USED}

This study used Sikament NN Superplasticizer. It is a superplasticizer liquid that functioned to reduce concrete's water to facilitate producing high initial strength and final strength, chlorine-free, and in accordance with ASTM C494-92. As superplasticizer it can increase workability, significantly reducing segregation risk, and normal stiffening time without retardation. Whereas as water-reducing material it can reduce water amount up to $20 \%$ which will increase compressive strength by $40 \%$ in 28 days. Typical dosage rate for use with the combination of manufactured sand/volcanic sand is $08, \%-2,3 \%$ by weight of cementitious material for norma precast concrete application. (PT. SIKA, 2016)

\subsection{TEST SPECIMEN}

Concrete sample testing was conducted when the concrete reaching 7, 14, and 28 days of age, with planned compressive strength by $20 \mathrm{Mpa}$. The test specimens were cylinder-shaped with $\mathrm{D}=150 \mathrm{~mm}$, and $\mathrm{T}=300 \mathrm{~mm}$. The quantity of the test specimens that had been made were 60 pcs with 15 samples for each mixture variable.

The test specimen samples that had been made consists of 4 types, they are :

1) Type I, a test specimen for normal concrete, were made as many as 15 samples, each testing used 5 samples at the age of 7,14 , and 28 days.

2) Type II, a test specimen for concrete with $1 \%$ superplasticizer addition without water reduction $(0 \%)$, were made as many as 15 samples, each testing used 5 samples at the age of 7, 14, and 28 days.

3) Type III, a test specimen for concrete with $1 \%$ superplasticizer addition and $15 \%$ water reduction, were made as many as 15 samples, each testing used 5 samples at the age of 7, 14, and 28 days.

4) Type IV, a test specimen for concrete with $1 \%$ superplasticizer addition and $17,5 \%$ water reduction , were made as many as 15 samples, each testing use 5 samples at the age of 7,14 , and 28 days.

\subsection{PROCEDURE OF STUDY}

Procedure of study was made to give description and simplify the process of study from the beginning to the analysis process and result evaluation. Began with preparing materials and tools, followed by material testing, concrete mixture K 250 planning, concrete mixture K 250 making, and then slump testing $(12 \pm 2 \mathrm{~cm})$.

If the slump testing did not meet the requirements, then back to concrete mixture making step. If the slump testing was succeeded, testing object was made, took care of, tested, and then the analysis and evaluation were carried out.

\subsection{CALCULATION OF CONCRETE COMPRESSIVE STRENGTH}

Concrete compressive strength is the amount of load for each unit area that causes concrete test specimen is destroyed if burdened with certain compressive strength that produced by compressing machine (SNI $031974-$ 1990). The maximum load mass result is legible in tons. The test specimen were placed/positioned on the machine's compressive board centrically. The loading process was slowly done until the concrete is destroyed. 
Analysis of The Effects of Superplasticizer Addition and Water Reduction in Concrete Mixture on Concrete

$\mathrm{fc}^{\prime}=\frac{P}{A}$

\section{Compressive Strength}

Note :

$\mathrm{fc}^{\prime}=$ Concrete Compressive Strength (Mpa)

$\mathrm{P}=$ Maximum Load Weight $(\mathrm{N})$

$\mathrm{A}=$ Test Specimen Surface Area $\left(\mathrm{mm}^{2}\right)$

\section{RESULTS AND DISCUSSIONS}

\subsection{FINE AGGREGATE GRADATION TEST}

Table 1: Results of Fine Aggregate Gradation Test

\begin{tabular}{|l|c|c|c|c|c|}
\hline Sieve & \multicolumn{2}{|c|}{ Weight Left Behind } & Cumulative Weight & Cumulative Weight of Passing \\
\hline Number & Mesh (mm) & (grams) & $(\%)$ & $(\%)$ & $(\%)$ \\
\hline $\mathbf{3 / 8 "}$ & 9,520 & 79,20 & 3,17 & 3,17 & 96,83 \\
\hline No. 4 & 4,750 & 314,80 & 12,60 & 15,77 & 84,23 \\
\hline No. 10 & 2,380 & 435,50 & 17,43 & 33,19 & 66,81 \\
\hline No.16 & 1,180 & 517,80 & 20,72 & 53,91 & 46,09 \\
\hline No.30 & 0,600 & 416,10 & 16,65 & 70,56 & 15,04 \\
\hline No.50 & 0,300 & 359,80 & 14,40 & 84,96 & 3,11 \\
\hline No.100 & 0,150 & 298,20 & 11,93 & 96,89 & 0,00 \\
\hline \multicolumn{7}{|c|}{ Rest } & 77,80 & 3,11 & & - \\
\hline Total & $2.499,20$ & 100,00 & 358,43 & \\
\hline
\end{tabular}

\subsection{COARSE AGGREGATE GRADATION TEST}

Table 2: Results of Coarse Aggregate Gradation Test

\begin{tabular}{|l|c|c|c|c|c|}
\hline Sieve & \multicolumn{2}{|c|}{ Weight Left Behind } & Cumulative Weight & Cumulative Weight of Passing \\
\hline Number & Mesh (mm) & (grams) & $(\%)$ & $(\%)$ & $(\%)$ \\
\hline $\mathbf{1 "}$ & 75,000 & - & 0,00 & 0,00 & 100,00 \\
\hline $\mathbf{3 / 4 "}$ & 38,000 & 70,00 & 2,13 & 2,13 & 97,87 \\
\hline $\mathbf{1 / 2 "}$ & 19,000 & $1.018,00$ & 30,96 & 33,08 & 66,92 \\
\hline $\mathbf{3 / 8 "}$ & 9,520 & 977,00 & 29,71 & 62,79 & 37,21 \\
\hline No.4 & 4,750 & 718,00 & 21,83 & 84,63 & 6,04 \\
\hline No.10 & 2,380 & 307,00 & 9,34 & 93,96 & 0,00 \\
\hline \multicolumn{7}{|c|}{ Rest } & 198,60 & 6,04 & - & - \\
\hline \multicolumn{7}{|c|}{ Total } & $3.288,60$ & 100,00 & 276,59 & \\
\hline
\end{tabular}

\subsection{UNIT WEIGHT}

The purpose of this examination was to find out the unit weight of sand and gravel that be used as mixture material for concrete production.

Table 3: Test Results of Serayu sand unit weight

\begin{tabular}{|l|l|c|c|c|}
\hline No. & Description & Unit & \multicolumn{2}{|c|}{ Measurement Result } \\
\hline $\mathbf{1}$ & Vessel Diameter & $\mathrm{Cm}$ & & 20,4 \\
\hline $\mathbf{2}$ & Vessel Height & $\mathrm{Cm}$ & & 5,0 \\
\hline $\mathbf{3}$ & Vessel Weight & grams & $\mathrm{A}$ & 287,2 \\
\hline $\mathbf{4}$ & Vessel Volume & $\mathrm{cm}^{3}$ & $\mathrm{~B}$ & $1.633,4$ \\
\hline
\end{tabular}


Dwi Sri Wiyanti, and Taufik Dwi Laksono

\begin{tabular}{|l|l|c|c|c|}
\hline $\mathbf{5}$ & Weight of Sand and Vessel & grams & C & $2.990,0$ \\
\hline $\mathbf{6}$ & Weight of Sand in the Vessel & grams & D = (C - A) & $2.702,8$ \\
\hline $\mathbf{7}$ & Unit Weight (shoveled) & grams $/ \mathrm{cm}^{3}$ & D/B & 1,655 \\
\hline
\end{tabular}

Table 4: Test Result of Gravel Unit Weight

\begin{tabular}{|l|l|c|c|c|}
\hline No. & Description & Unit & \multicolumn{2}{c|}{ Measurement Result } \\
\hline $\mathbf{1}$ & Vessel Diameter & $\mathrm{Cm}$ & & 20,4 \\
\hline $\mathbf{2}$ & Vessel Height & $\mathrm{Cm}$ & & 5,0 \\
\hline $\mathbf{3}$ & Vessel Weight & $\mathrm{Grams}$ & $\mathrm{A}$ & 287,2 \\
\hline $\mathbf{4}$ & Vessel Volume & $\mathrm{cm}^{3}$ & $\mathrm{~B}$ & $1.633,4$ \\
\hline $\mathbf{5}$ & Weight of Sand and Vessel & $\mathrm{Grams}$ & $\mathrm{C}$ & $2.552,0$ \\
\hline $\mathbf{6}$ & Weight of Sand in the Vessel & Grams & D = (C - A) & $2.264,8$ \\
\hline $\mathbf{7}$ & Unit Weight (shoveled) & grams $/ \mathrm{cm}^{3}$ & D/B & 1,387 \\
\hline
\end{tabular}

\subsection{DENSITY}

Density examination was conducted to determine the dry density of the saturated surface of fine and coarse aggregate to find out the quality of the sand and gravel that will be used.

Table 5: Test Results of Serayu Sand Density

\begin{tabular}{|l|l|c|c|c|}
\hline No. & Description & Unit & Sample 1 & Sample 2 \\
\hline $\mathbf{1}$ & Weight of piknometer + sand + water (B1) & (gr) & 752,5 & 754,0 \\
\hline $\mathbf{2}$ & Weight of stove dry sand (B2) & (gr) & 477,5 & 475 \\
\hline $\mathbf{3}$ & Weight of water filled piknometer (B3) & (gr) & 450,2 & 452 \\
\hline $\mathbf{4}$ & Weight of surface sand dry /SSD (B4) & (gr) & 500,0 & 500,0 \\
\hline $\mathbf{5}$ & Density = B2/(B3 + B4 - B1) & & \multicolumn{3}{|c|}{2,41} \\
\hline $\mathbf{6}$ & Average Density & & \multicolumn{3}{|c|}{2,53} & 2,53 \\
\hline $\mathbf{7}$ & SSD Density = B4/ (B3 + B4 - B1) & & \multicolumn{3}{|c|}{4,93} \\
\hline $\mathbf{8}$ & Average Density of SSD & (\%) & 4,71 & 5,26 \\
\hline $\mathbf{9}$ & Water Absorbtion & (\%) & \multicolumn{3}{|c|}{4,99} \\
\hline $\mathbf{1 0}$ & Average Water Absorbtion & \multicolumn{3}{|c|}{} \\
\hline
\end{tabular}

Table 6: Test Results of Gravel Density

\begin{tabular}{|l|l|c|c|c|}
\hline No. & Description & Unit & Sample 1 & Sample 2 \\
\hline $\mathbf{1}$ & Gravel Weight after roasting (A) & (gr) & $2.939,8$ & $2.941,7$ \\
\hline $\mathbf{2}$ & Gravel weight in water (B) & (gr) & $1.752,6$ & $1.759,5$ \\
\hline $\mathbf{3}$ & Gravel weight in SSD condition (C) & (gr) & $3.000,0$ & $3.000,0$ \\
\hline $\mathbf{4}$ & Density = A/C - B) & & 2,36 & 2,37 \\
\hline $\mathbf{5}$ & Average Density & & \multicolumn{3}{|c|}{2,36} \\
\hline $\mathbf{6}$ & SSD Density = C/ (C - B) & & \multicolumn{3}{|c|}{2,41} \\
\hline $\mathbf{7}$ & Average SSD Density & (\%) & \multicolumn{3}{|c|}{2,42} \\
\hline $\mathbf{8}$ & Water Absorption = (C - A)/A x 100\% & (\%) & \multicolumn{3}{|c|}{2,05} & 1,98 \\
\hline $\mathbf{9}$ & Average water absorption & \multicolumn{4}{|c|}{} \\
\hline
\end{tabular}

\subsection{MUD LEVEL}

Mud level test is needed to find out the amount of mud in fine aggregate

Table 7: Test Results of Serayu Sand Mud Level

\begin{tabular}{|l|l|c|c|c|}
\hline No. & Description & Unit & Sample I & Sample II \\
\hline $\mathbf{1}$ & Weight of dry aggregate (beginning) + cup & (gr) & 95,46 & 88,55 \\
\hline $\mathbf{2}$ & Weight of dry aggregate (final) + cup & (gr) & 93,01 & 85,17 \\
\hline
\end{tabular}


Analysis of The Effects of Superplasticizer Addition and Water Reduction in Concrete Mixture on Concrete Compressive Strength

\begin{tabular}{|c|c|c|c|c|c|c|}
\hline 3 & \multicolumn{3}{|l|}{ Weight of Cup } & (gr) & 17,28 & 17,66 \\
\hline 4 & \multicolumn{3}{|c|}{ Weight of dry aggregate (beginning) (A) } & (gr) & 78,18 & 70,89 \\
\hline 5 & \multicolumn{3}{|c|}{ Weight of dry aggregate (final) (B) } & (gr) & 75,73 & 67,51 \\
\hline \multirow[t]{2}{*}{6} & \multirow[t]{2}{*}{ Mud Level = } & $(A-B)$ & \multirow[t]{2}{*}{ x $100 \%$} & $(\%)$ & \multirow[t]{2}{*}{3,13} & \multirow{2}{*}{4,77} \\
\hline & & $\mathrm{A}$ & & & & \\
\hline 7 & \multicolumn{3}{|c|}{ Average Mud Level } & $(\%)$ & \multicolumn{2}{|c|}{3,95} \\
\hline
\end{tabular}

\subsection{COMPOSITION OF NORMAL CONCRETE MIXTURE}

Based on the material test results, in accordance with Mix Design SK.SNI. T-15-1990-03, the obtained normal concrete mixture for 20 Mpa compressive strength were:

Table 8: Material Requirements for normal concrete mixture per $\mathrm{m}^{3}$

\begin{tabular}{|c|l|c|c|}
\hline 1. & Water & $\mathbf{2 2 5}$ & liter \\
\hline 2. & Cement & 460 & $\mathrm{~kg}$ \\
\hline 3. & Fine Aggregate (Sand) & 594 & $\mathrm{Kg}$ \\
\hline 4. & Coarse Aggregate (Gravel) & 1105 & $\mathrm{Kg}$ \\
\hline & Total & 2384 & $\mathrm{Kg}$ \\
\hline
\end{tabular}

Composition of Concrete Mixture with Superplasticizer and Water Reduction

This study was planned to make as many as 60 concrete samples with several types as follows :

Table 9: Type I : Concrete Mix Design per- m3 (normal concrete without superplasticizer)

\begin{tabular}{|c|l|c|c|}
\hline 1. & Water & $\mathbf{2 2 5}$ & Liter \\
\hline 2. & Cement & 460 & $\mathrm{Kg}$ \\
\hline 3. & Fine Aggregate (Sand) & 594 & $\mathrm{Kg}$ \\
\hline 4. & Coarse Aggregate (Gravel) & 1105 & $\mathrm{Kg}$ \\
\hline 5. & Superplasticizer & $0 \%$ & \\
\hline
\end{tabular}

Table 10: Type II: Concrete Mix Design per- $\mathrm{m}^{3}$ (concrete with $1 \%$ superplasticizer)

\begin{tabular}{|c|l|c|c|}
\hline 1. & Water & $\mathbf{2 2 5}$ & liter \\
\hline 2. & Cement & 460 & $\mathrm{~kg}$ \\
\hline 3. & Fine Aggregate (Sand) & 594 & $\mathrm{~kg}$ \\
\hline 4. & Coarse Aggregate (Gravel) & 1105 & $\mathrm{~kg}$ \\
\hline 5. & Superplasticizer & $1 \%$ & \\
\hline
\end{tabular}

Table 11: Type III: Concrete Mix Design per- $\mathrm{m}^{3}$ (Concrete with 1\% Superplasticizer and 15\% water reduction)

\begin{tabular}{|c|l|c|c|}
\hline 1. & Water & $\mathbf{1 9 1 , 2 5}$ & Liter \\
\hline 2. & Cement & 460 & $\mathrm{Kg}$ \\
\hline 3. & Fine Aggregate (Sand) & 594 & $\mathrm{Kg}$ \\
\hline 4. & Coarse Agregat (Gravel) & 1105 & $\mathrm{Kg}$ \\
\hline 5. & Superplasticizer & $1 \%$ & \\
\hline
\end{tabular}

Table 12: Type IV: Concrete Mix Design per- m3 (Concrete with 1\% Superplasticizer and 17,5\% water reduction)

\begin{tabular}{|c|l|c|c|}
\hline 1. & Water & $\mathbf{1 8 5 , 6 2 5}$ & liter \\
\hline 2. & Cement & 460 & $\mathrm{Kg}$ \\
\hline 3. & Fine Aggregate (Sand) & 594 & $\mathrm{Kg}$ \\
\hline 4. & Coarse Agregat (Gravel) & 1105 & $\mathrm{Kg}$ \\
\hline 5. & Superplasticizer & $1 \%$ & \\
\hline
\end{tabular}




\subsection{THE PRODUCED CONCRETE COMPRESSIVE STRENGTH}

Dwi Sri Wiyanti, and Taufik Dwi Laksono

Based on 4 type of concrete mixture, the obtained results of concrete compressive strength test were :

Table 13: Normal Concrete age of 7 days

\begin{tabular}{|c|c|c|c|c|c|c|c|c|}
\hline \multirow[t]{4}{*}{ NO } & Code & \multirow{4}{*}{$\begin{array}{c}\text { Dimension } \\
\text { Cylinder } \\
(\mathrm{cm})\end{array}$} & \multirow{4}{*}{$\begin{array}{l}\text { Weight } \\
\text { (grams) }\end{array}$} & \multirow{4}{*}{$\begin{array}{l}\text { Area } \\
(\mathrm{cm})\end{array}$} & \multirow{4}{*}{$\begin{array}{c}\text { Load } \\
\operatorname{Max}(\mathrm{N})\end{array}$} & \multicolumn{2}{|c|}{ Age of 7 days } & \multirow{3}{*}{$\begin{array}{c}\text { Age Review } \\
28 \text { days } \\
\text { f'cu }\end{array}$} \\
\hline & & & & & & & & \\
\hline & \multirow[t]{2}{*}{ Test Specimen } & & & & & $\mathrm{K}$ & \multirow[t]{2}{*}{ f'c } & \\
\hline & & & & & & $(\mathrm{kg} / \mathrm{cm} 2)$ & & \\
\hline 1 & $\mathrm{~N}-7$ & $15 \times 30$ & 12306 & 176,71 & 290000 & 167,29 & 13,89 & 19,84 \\
\hline 2 & $\mathrm{~N}-7$ & $15 \times 30$ & 12299 & 176,71 & 310000 & 178,83 & 14,84 & 21,20 \\
\hline 3 & $\mathrm{~N}-7$ & $15 \times 30$ & 12317 & 176,71 & 290000 & 167,29 & 13,89 & 19,84 \\
\hline 4 & $\mathrm{~N}-7$ & $15 \times 30$ & 12294 & 176,71 & 300000 & 173,06 & 14,36 & 20,52 \\
\hline 5 & $\mathrm{~N}-7$ & $15 \times 30$ & 12310 & 176,71 & 320000 & 184,59 & 15,32 & 21,89 \\
\hline \multicolumn{6}{|c|}{ Average } & 174,21 & 14,46 & 20,66 \\
\hline
\end{tabular}

Table 14: 1\% Superplasticizer Admixture Concrete age of 7 days

\begin{tabular}{|c|c|c|c|c|c|c|c|c|}
\hline \multirow[t]{3}{*}{ NO } & Code & \multirow[t]{2}{*}{ Dimension } & Weight & Area & Load & \multicolumn{2}{|c|}{ Age of 7 days } & \multirow{2}{*}{$\begin{array}{c}\text { Age Review } \\
28 \text { days }\end{array}$} \\
\hline & \multirow{2}{*}{ Test Specimen } & & \multirow{2}{*}{ (grams) } & \multirow{2}{*}{$(\mathrm{cm})$} & \multirow{2}{*}{$\operatorname{Max}(\mathrm{N})$} & $\mathrm{K}$ & \multirow{2}{*}{$\mathrm{f}^{\prime} \mathrm{c}$} & \\
\hline & & $(\mathrm{cm})$ & & & & $(\mathrm{kg} / \mathrm{cm} 2)$ & & \\
\hline 1 & $1 \%-7$ & $15 \times 30$ & 12411 & 176,71 & 320000 & 184,59 & 15,32 & 21,89 \\
\hline 2 & $1 \%-7$ & $15 \times 30$ & 12373 & 176,71 & 300000 & 173,06 & 14,36 & 20,52 \\
\hline 3 & $1 \%-7$ & $15 \times 30$ & 12473 & 176,71 & 320000 & 184,59 & 15,32 & 21,89 \\
\hline 4 & $1 \%-7$ & $15 \times 30$ & 12575 & 176,71 & 300000 & 173,06 & 14,36 & 20,52 \\
\hline 5 & $1 \%-7$ & $15 \times 30$ & 12418 & 176,71 & 290000 & 167,29 & 13,89 & 19,84 \\
\hline & & Averag & & & & 176,52 & 14,65 & 20,93 \\
\hline
\end{tabular}

Table 15: 1\% Superplasticizer Admixture and 15\% Water Reduction Concrete age of 7 days

\begin{tabular}{|c|c|c|c|c|c|c|c|c|}
\hline \multirow[t]{4}{*}{ NO } & Code & Dimension & Weight & Area & Load & \multicolumn{2}{|c|}{ Age of 7 days } & \multirow{4}{*}{$\begin{array}{c}\text { Age Review } \\
28 \text { days } \\
\text { f'cu }\end{array}$} \\
\hline & & & & & & & & \\
\hline & \multirow[t]{2}{*}{ Test Specimen } & Cylinder & \multirow[t]{2}{*}{ (grams) } & \multirow[t]{2}{*}{$(\mathrm{cm})$} & \multirow[t]{2}{*}{$\operatorname{Max}(N)$} & $\mathrm{K}$ & \multirow[t]{2}{*}{$\mathrm{f}^{\prime} \mathrm{C}$} & \\
\hline & & $(\mathrm{cm})$ & & & & $(\mathrm{kg} / \mathrm{cm} 2)$ & & \\
\hline 1 & $1 \%, 15 \%-7$ & $15 \times 30$ & 12502 & 176,71 & 320000 & 184,59 & 15,32 & 21,89 \\
\hline 2 & $1 \%, 15 \%-7$ & $15 \times 30$ & 12696 & 176,71 & 310000 & 178,83 & 14,84 & 21,20 \\
\hline 3 & $1 \%, 15 \%-7$ & $15 \times 30$ & 12437 & 176,71 & 310000 & 178,83 & 14,84 & 21,20 \\
\hline 4 & $1 \%, 15 \%-7$ & $15 \times 30$ & 12648 & 176,71 & 300000 & 173,06 & 14,36 & 20,52 \\
\hline 5 & $1 \%, 15 \%-7$ & $15 \times 30$ & 12540 & 176,71 & 300000 & 173,06 & 14,36 & 20,52 \\
\hline \multicolumn{6}{|c|}{ Average } & 177,67 & 14,75 & 21,07 \\
\hline
\end{tabular}

Table 16: 1\% Superplasticizer Admixture and 17,5\% Water Reduction Concrete age of 7 days

\begin{tabular}{|c|c|c|c|c|c|c|c|c|}
\hline \multirow[t]{4}{*}{ NO } & Code & \multirow{2}{*}{ Dimension } & \multirow[t]{2}{*}{ Weight } & \multirow[t]{2}{*}{ Area } & \multirow[t]{2}{*}{ Load } & \multicolumn{2}{|c|}{ Age of 7 days } & \multirow{2}{*}{$\begin{array}{c}\text { Age Review } \\
28 \text { days }\end{array}$} \\
\hline & & & & & & & & \\
\hline & \multirow[t]{2}{*}{ Test Specimen } & Cylinder & \multirow[t]{2}{*}{ (grams) } & \multirow[t]{2}{*}{$(\mathrm{cm})$} & \multirow[t]{2}{*}{$\operatorname{Max}(N)$} & $\mathrm{K}$ & \multirow[t]{2}{*}{$\mathrm{f}^{\prime} \mathrm{c}$} & \multirow[t]{2}{*}{$\mathrm{f}^{\prime} \mathrm{cu}$} \\
\hline & & $(\mathrm{cm})$ & & & & $(\mathrm{kg} / \mathrm{cm} 2)$ & & \\
\hline 1 & $1 \%, 17,5 \%-7$ & $15 \times 30$ & 12608 & 176,71 & 280000 & 161,52 & 13,41 & 19,15 \\
\hline 2 & $1 \%, 17,5 \%-7$ & $15 \times 30$ & 12357 & 176,71 & 270000 & 155,75 & 12,93 & 18,47 \\
\hline 3 & $1 \%, 17,5 \%-7$ & $15 \times 30$ & 12590 & 176,71 & 280000 & 161,52 & 13,41 & 19,15 \\
\hline 4 & $1 \%, 17,5 \%-7$ & $15 \times 30$ & 12422 & 176,71 & 300000 & 173,06 & 14,36 & 20,52 \\
\hline 5 & $1 \%, 17,5 \%-7$ & $15 \times 30$ & 12393 & 176,71 & 280000 & 161,52 & 13,41 & 19,15 \\
\hline \multicolumn{6}{|c|}{ Average } & 162,67 & 13,50 & 19,29 \\
\hline
\end{tabular}


Analysis of The Effects of Superplasticizer Addition and Water Reduction in Concrete Mixture on Concrete Compressive Strength

Table 17: Normal Concrete Age of 14 days

\begin{tabular}{|c|c|c|c|c|c|c|c|c|}
\hline \multirow[t]{4}{*}{ NO } & Code & \multirow{4}{*}{$\begin{array}{c}\text { Dimension } \\
\text { Cylinder } \\
(\mathrm{cm})\end{array}$} & \multirow{4}{*}{$\begin{array}{l}\text { Weight } \\
\text { (grams) }\end{array}$} & \multirow{4}{*}{$\begin{array}{l}\text { Area } \\
(\mathrm{cm})\end{array}$} & \multirow{4}{*}{$\begin{array}{c}\text { Load } \\
\operatorname{Max}(N)\end{array}$} & \multicolumn{2}{|c|}{ Age of 14 days } & \multirow{4}{*}{$\begin{array}{c}\text { Age Review } \\
28 \text { days } \\
\text { f'cu }\end{array}$} \\
\hline & & & & & & & & \\
\hline & \multirow[t]{2}{*}{ Test Specimen } & & & & & $\mathrm{K}$ & \multirow[t]{2}{*}{$\mathrm{f}^{\prime} \mathrm{c}$} & \\
\hline & & & & & & $(\mathrm{kg} / \mathrm{cm} 2)$ & & \\
\hline 1 & $\mathrm{~N}-14$ & $15 \times 30$ & 12318 & 176,71 & 390000 & 224,98 & 18,67 & 21,22 \\
\hline 2 & $\mathrm{~N}-14$ & $15 \times 30$ & 12311 & 176,71 & 380000 & 219,21 & 18,19 & 20,68 \\
\hline 3 & $\mathrm{~N}-14$ & $15 \times 30$ & 12293 & 176,71 & 400000 & 230,74 & 19,15 & 21,76 \\
\hline 4 & $\mathrm{~N}-14$ & $15 \times 30$ & 12301 & 176,71 & 390000 & 224,98 & 18,67 & 21,22 \\
\hline 5 & $\mathrm{~N}-14$ & $15 \times 30$ & 12490 & 176,71 & 380000 & 219,21 & 18,19 & 20,68 \\
\hline \multicolumn{6}{|c|}{ Average } & 223,82 & 18,58 & 21,11 \\
\hline
\end{tabular}

Table 18: 1\% Superplasticizer Admixture Concrete age of 14 days

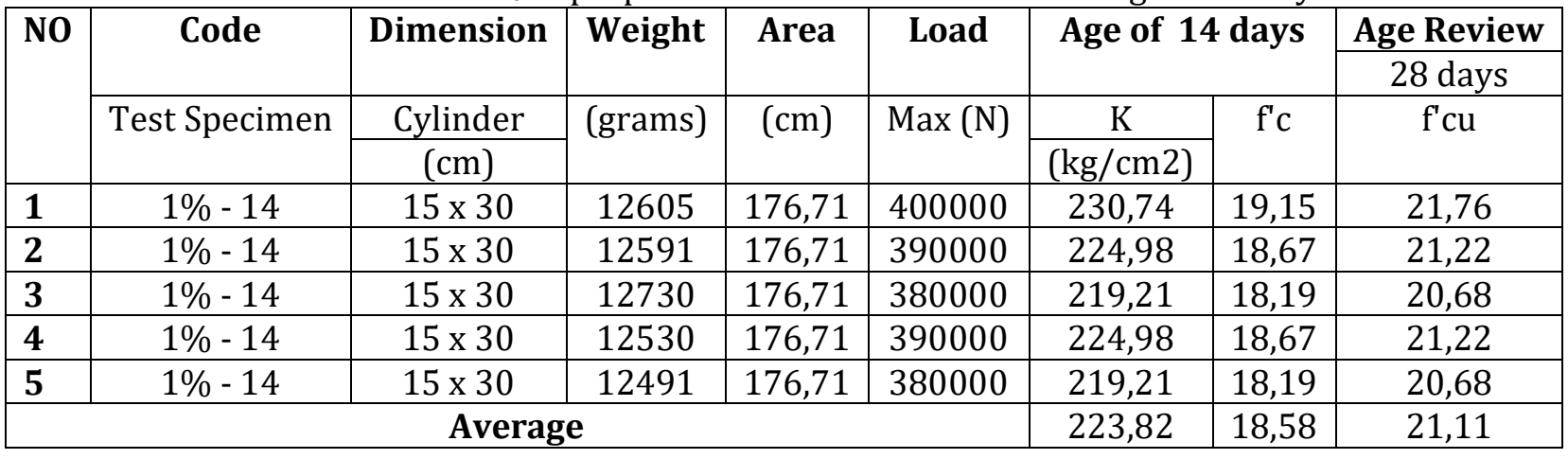

Table 19: 1\% Superplasticizer Admixture and 15\% Water Reduction Concrete age of 14 days

\begin{tabular}{|c|c|c|c|c|c|c|c|c|}
\hline \multirow[t]{4}{*}{ NO } & \multirow[t]{2}{*}{ Code } & \multirow[t]{2}{*}{ Dimension } & \multirow[t]{2}{*}{ Weight } & \multirow[t]{2}{*}{ Area } & \multirow[t]{2}{*}{ Load } & \multicolumn{2}{|c|}{ Age of 14 days } & \multirow{2}{*}{$\begin{array}{c}\text { Age Review } \\
28 \text { days }\end{array}$} \\
\hline & & & & & & & & \\
\hline & \multirow[t]{2}{*}{ Test Specimen } & Cylinder & \multirow[t]{2}{*}{ (grams) } & \multirow[t]{2}{*}{$(\mathrm{cm})$} & \multirow[t]{2}{*}{$\operatorname{Max}(N)$} & $\mathrm{K}$ & \multirow[t]{2}{*}{$\mathrm{f}^{\prime} \mathrm{c}$} & \multirow[t]{2}{*}{$\mathrm{f}^{\prime} \mathrm{cu}$} \\
\hline & & $(\mathrm{cm})$ & & & & $(\mathrm{kg} / \mathrm{cm} 2)$ & & \\
\hline 1 & $1 \%, 15 \%-14$ & $15 \times 30$ & 12581 & 176,71 & 380000 & 219,21 & 18,19 & 20,68 \\
\hline 2 & $15 \%-14$ & $15 \times 30$ & 12469 & 176,71 & 400000 & 230,74 & 19,15 & 21,76 \\
\hline 3 & $1 \%, 15 \%-14$ & $15 \times 30$ & 12680 & 176,71 & 390000 & 224,98 & 18,67 & 21,22 \\
\hline 4 & $1 \%, 15 \%-14$ & $15 \times 30$ & 12488 & 176,71 & 380000 & 219,21 & 18,19 & 20,68 \\
\hline 5 & $1 \%, 15 \%-14$ & $15 \times 30$ & 12505 & 176,71 & 400000 & 230,74 & 19,15 & 21,76 \\
\hline \multicolumn{6}{|c|}{ Average } & 224,98 & 18,67 & 21,22 \\
\hline
\end{tabular}

Table 20: 1\% Superplasticizer Admixture and 17,5 \% Water Reduction Concrete age of 14 days

\begin{tabular}{|c|c|c|c|c|c|c|c|c|}
\hline \multirow[t]{4}{*}{ NO } & \multirow[t]{2}{*}{ Code } & \multirow[t]{2}{*}{ Dimension } & \multirow[t]{2}{*}{ Weight } & \multirow[t]{2}{*}{ Area } & \multirow[t]{2}{*}{ Load } & \multicolumn{2}{|c|}{ Age of 14 days } & \multirow{2}{*}{$\begin{array}{l}\text { Age Review } \\
28 \text { days }\end{array}$} \\
\hline & & & & & & & & \\
\hline & \multirow[t]{2}{*}{ Test Specimen } & Cylinder & \multirow[t]{2}{*}{ (grams) } & \multirow[t]{2}{*}{$(\mathrm{cm})$} & \multirow[t]{2}{*}{$\operatorname{Max}(N)$} & $\mathrm{K}$ & \multirow[t]{2}{*}{$\mathrm{f}^{\prime} \mathrm{c}$} & \multirow[t]{2}{*}{$\mathrm{f}^{\prime} \mathrm{cu}$} \\
\hline & & $(\mathrm{cm})$ & & & & $(\mathrm{kg} / \mathrm{cm} 2)$ & & \\
\hline 1 & $1 \%, 17,5 \%-14$ & $15 \times 30$ & 12489 & 176,71 & 360000 & 207,67 & 17,24 & 19,59 \\
\hline 2 & $1 \%, 17,5 \%-14$ & $15 \times 30$ & 12832 & 176,71 & 350000 & 201,90 & 16,76 & 19,04 \\
\hline 3 & $1 \%, 17,5 \%-14$ & $15 \times 30$ & 12555 & 176,71 & 340000 & 196,13 & 16,28 & 18,50 \\
\hline 4 & $1 \%, 17,5 \%-14$ & $15 \times 30$ & 12702 & 176,71 & 360000 & 207,67 & 17,24 & 19,59 \\
\hline 5 & $17,5 \%-14$ & $15 \times 30$ & 12522 & 176,71 & 370000 & 213,44 & 17,72 & 20,13 \\
\hline \multicolumn{6}{|c|}{ Average } & 205,36 & 17,05 & 19,37 \\
\hline
\end{tabular}


Table 21: Normal Concrete Age of 28 days

\begin{tabular}{|c|c|c|c|c|c|c|c|c|}
\hline \multirow[t]{3}{*}{ NO } & Code & Dimension & Weight & Area & Load & \multicolumn{3}{|c|}{$\begin{array}{c}\text { Age Review } \\
28 \text { days }\end{array}$} \\
\hline & \multirow[t]{2}{*}{ Test Specimen } & Cylinder & \multirow[t]{2}{*}{ (grams) } & \multirow[t]{2}{*}{$(\mathrm{cm})$} & \multirow[t]{2}{*}{$\operatorname{Max}(\mathrm{N})$} & $\mathrm{K}$ & \multirow[t]{2}{*}{$\mathrm{f}^{\prime} \mathrm{c}$} & \multirow[t]{2}{*}{$\mathrm{f}^{\prime} \mathrm{cu}$} \\
\hline & & $(\mathrm{cm})$ & & & & $(\mathrm{kg} / \mathrm{cm} 2)$ & & \\
\hline 1 & $N-28$ & $15 \times 30$ & 12540 & 176,71 & 440000 & 253,82 & 21,07 & 21,07 \\
\hline 2 & 28 & & 126 & 176,71 & 450000 & 259,59 & 21,55 & 21,55 \\
\hline 3 & $\mathrm{~N}-28$ & $15 \times 30$ & 12498 & 176,71 & 470000 & 271,12 & 22,50 & 22,50 \\
\hline 4 & -28 & $15 \times 30$ & 12512 & 176,71 & 430000 & 248,05 & 20,59 & 20,59 \\
\hline 5 & $\mathrm{~N}-28$ & $15 \times 30$ & 12490 & 176,71 & 470000 & 271,12 & 22,50 & 22,50 \\
\hline \multicolumn{6}{|c|}{ Average } & 205,36 & 17,05 & 19,37 \\
\hline
\end{tabular}

Table 22: 1\% Superplasticizer Admixture Concrete age of 28 days

\begin{tabular}{|c|c|c|c|c|c|c|c|c|}
\hline \multirow[t]{3}{*}{ NO } & Code & Dimension & Weight & Area & Load & \multicolumn{3}{|c|}{$\begin{array}{c}\text { Age Review } \\
28 \text { days }\end{array}$} \\
\hline & \multirow[t]{2}{*}{ Test Specimen } & Cylinder & \multirow[t]{2}{*}{ (grams) } & \multirow[t]{2}{*}{$(\mathrm{cm})$} & \multirow[t]{2}{*}{$\operatorname{Max}(\mathrm{N})$} & $\mathrm{K}$ & \multirow[t]{2}{*}{$\mathrm{f}^{\prime} \mathrm{c}$} & \multirow[t]{2}{*}{$\mathrm{f}^{\prime} \mathrm{cu}$} \\
\hline & & $(\mathrm{cm})$ & & & & $(\mathrm{kg} / \mathrm{cm} 2)$ & & \\
\hline 1 & $1 \%-28$ & $15 \times 30$ & 12470 & 176,71 & 450000 & 259,59 & 21,55 & 21,55 \\
\hline 2 & $1 \%-28$ & $5 \times 30$ & 12669 & 176,71 & 510000 & 294,20 & 24,42 & 24,42 \\
\hline 3 & $1 \%-28$ & $15 \times 30$ & 12497 & 176,71 & 440000 & 253,82 & 21,07 & 21,07 \\
\hline 4 & $1 \%-28$ & $5 \times 30$ & 12705 & 176,71 & 430000 & 248,05 & 20,59 & 20,59 \\
\hline 5 & $1 \%-28$ & $15 \times 30$ & 12490 & 176,71 & 470000 & 271,12 & 22,50 & 22,50 \\
\hline \multicolumn{6}{|c|}{ Average } & 205,36 & 17,05 & 19,37 \\
\hline
\end{tabular}

Table 23: 1\% Superplasticizer Admixture and 15\% Water Reduction Concrete age of 28 days

\begin{tabular}{|c|c|c|c|c|c|c|c|c|}
\hline \multirow[t]{3}{*}{ NO } & Code & Dimension & Weight & Area & Load & \multicolumn{3}{|c|}{$\begin{array}{c}\text { Age Review } \\
28 \text { days }\end{array}$} \\
\hline & \multirow[t]{2}{*}{ Test Specimen } & Cylinder & \multirow[t]{2}{*}{ (grams) } & \multirow[t]{2}{*}{$(\mathrm{cm})$} & \multirow[t]{2}{*}{$\operatorname{Max}(\mathrm{N})$} & $\mathrm{K}$ & \multirow[t]{2}{*}{$\mathrm{f}^{\prime} \mathrm{c}$} & \multirow[t]{2}{*}{$\mathrm{f}^{\prime} \mathrm{cu}$} \\
\hline & & $(\mathrm{cm})$ & & & & $(\mathrm{kg} / \mathrm{cm} 2)$ & & \\
\hline 1 & $1 \%, 15 \%-28$ & $15 \times 30$ & 12490 & 176,71 & 490000 & 282,66 & 23,46 & 23,46 \\
\hline 2 & $1 \%, 15 \%-28$ & $15 \times 30$ & 12745 & 176,71 & 470000 & 271,12 & 22,50 & 22,50 \\
\hline 3 & $1 \%, 15 \%-28$ & $15 \times 30$ & 12571 & 176,71 & 510000 & 294,20 & 24,42 & 24,42 \\
\hline 4 & $1 \%, 15 \%-28$ & $15 \times 30$ & 12420 & 176,71 & 430000 & 248,05 & 20,59 & 20,59 \\
\hline 5 & $1 \%, 15 \%-28$ & $15 \times 30$ & 12446 & 176,71 & 500000 & 288,43 & 23,94 & 23,94 \\
\hline \multicolumn{6}{|c|}{ Average } & 205,36 & 17,05 & 19,37 \\
\hline
\end{tabular}

Table 24: 1\% Superplasticizer Admixture and 17,5 \% Water Reduction Concrete age of 28 days

\begin{tabular}{|c|c|c|c|c|c|c|c|c|}
\hline \multirow[t]{3}{*}{ NO } & Code & Dimension & Weight & Area & Load & \multicolumn{3}{|c|}{$\begin{array}{c}\text { Age Review } \\
28 \text { days }\end{array}$} \\
\hline & \multirow[t]{2}{*}{ Test Specimen } & Cylinder & \multirow[t]{2}{*}{ (grams) } & \multirow[t]{2}{*}{$(\mathrm{cm})$} & \multirow[t]{2}{*}{$\operatorname{Max}(N)$} & $\mathrm{K}$ & \multirow[t]{2}{*}{$f^{\prime} c$} & \multirow[t]{2}{*}{$\mathrm{f}^{\prime} \mathrm{cu}$} \\
\hline & & $(\mathrm{cm})$ & & & & $(\mathrm{kg} / \mathrm{cm} 2)$ & & \\
\hline 1 & $1 \%, 15 \%-28$ & $15 \times 30$ & 12332 & 176,71 & 410000 & 236,51 & 19,63 & 19,63 \\
\hline 2 & $1 \%, 15 \%-28$ & $15 \times 30$ & 12449 & 176,71 & 410000 & 236,51 & 19,63 & 19,63 \\
\hline 3 & $1 \%, 15 \%-28$ & $15 \times 30$ & 12510 & 176,71 & 400000 & 230,74 & 19,15 & 19,15 \\
\hline 4 & $1 \%, 15 \%-28$ & $15 \times 30$ & 12653 & 176,71 & 420000 & 242,28 & 20,11 & 20,11 \\
\hline 5 & $1 \%, 15 \%-28$ & $15 \times 30$ & 12519 & 176,71 & 410000 & 236,51 & 19,63 & 19,63 \\
\hline \multicolumn{6}{|c|}{ Average } & 205,36 & 17,05 & 19,37 \\
\hline
\end{tabular}


Analysis of The Effects of Superplasticizer Addition and Water Reduction in Concrete Mixture on Concrete

\subsection{DISCUSSION}

\section{Compressive Strength} follows :

Based on the obtained compressive strength of each type and the duration of testing, a table can be made as

Table 25: Test Result Recapitulation for each type and test duration

\begin{tabular}{|l|l|c|c|c|c|c|c|}
\hline No & Type & \multicolumn{4}{|c|}{ Average Compressive Strength Based On The Age Of Test Specimen } \\
\cline { 2 - 7 } & $\begin{array}{c}7 \\
\text { Days }\end{array}$ & $\begin{array}{c}28 \text { days } \\
\text { Conversion }\end{array}$ & $\begin{array}{c}14 \\
\text { Days }\end{array}$ & $\begin{array}{c}14 \text { days } \\
\text { Conversion }\end{array}$ & $\begin{array}{c}28 \\
\text { Days }\end{array}$ & $\begin{array}{c}28 \text { Days } \\
\text { Conversion }\end{array}$ \\
\hline $\mathbf{1}$ & I (Normal Concrete) & 14,46 & 20,66 & 18,58 & 21,11 & 21,64 & 21,64 \\
\hline $\mathbf{2}$ & $\begin{array}{l}\text { II (Concrete with 1\% } \\
\text { superplasticizer) }\end{array}$ & 14,65 & 20,93 & 18,58 & 21,11 & 22,02 & 22,02 \\
\hline $\mathbf{3}$ & $\begin{array}{l}\text { III (Concrete with 1\% } \\
\text { superplasticizer and 15\% water } \\
\text { reduction) }\end{array}$ & 14,75 & 21,07 & 18,67 & 21,22 & 22,98 & 22,98 \\
\hline $\mathbf{4}$ & $\begin{array}{l}\text { IV (Concrete with 1\% } \\
\text { superplasticizer and 17,5\% } \\
\text { water reduction) }\end{array}$ & 13,50 & 19,29 & 17,05 & 19,37 & 19,63 & 19,63 \\
\hline
\end{tabular}

Based on table 25, it can be seen that each type have different compressive strength. It can be seen that Type III concrete compressive strength which is concrete with $1 \%$ superplasticizer and $15 \%$ water reduction have the most optimum 28 days of age concrete compressive strength that is 22,98 Mpa. Whereas Type IV concrete whish is concrete concrete with $1 \%$ superplasticizer and 17,5\% water reduction at 7, 14, and 28 days of age testing have lower compressive strength than Type I concrete or normal concrete. So, it can be said that concrete addition with $1 \%$ superplasticizer and $17,5 \%$ water reduction will produce lower concrete compressive strength compared to normal concrete compressive strength.

\section{CONCLUSIONS AND RECOMMENDATIONS}

Based on research results and discussion, it can be concluded that superplasticizer addition with a certain dose or percentage towards cement weight and water amount reduction in concrete mixture can increase concrete compressive strength. As for water reduction, $15 \%$ of water reduction is the maximum amount to produce optimum compressive strength because $17,5 \%$ of water reduction produced lower concrete compressive strength than normal concrete compressive strength.

The increase or decrease in produced concrete compressive strength is not only fully influenced by superplasticizer addition or water reduction in concrete's mixture but also influenced by fine aggregate and coarse aggregate materials that be used.

\section{SOURCES OF FUNDING}

None.

\section{CONFLICT OF INTEREST}

None.

\section{ACKNOWLEDGMENT}

None. 


\section{REFERENCES}

[1] Ali Asroni, 2010. Balok dan Plat Beton Bertulang. Graha Ilmu, Yogyakarta

[2] Ali Hussein Hameed. 2012. Effect of Superplasticizer Dosage on Workability of Self Compact Concrete. Diyala Journal of Engineering Sciences, Vol. 05, No. 02, pp 66 - 81, December 2012.

[3] Asrullah, 2019. Analisa Kuat Tekan Beton Dengan Menggunakan Sika Concrete Refair Mortar Dan Tempurung Kelapa Pada Campuran Beton K300. Jurnal Teknik Sipil UNPAL, Vol. 9, No. 1, Mei 2019

[4] Departemen Pekerjaan Umum, 1990, Metode Pengujian Kuat Tekan Beton Berdasarkan SNI 03-1974-1990. Badan Standarisasi Nasional

[5] Diarto Trisnoyuwono, 2014. Beton Non Pasir, Graha Ilmu. Yogyakarta

[6] M. Benaicha, A. Hafidi Alaoui, O. Jalbaud, Y. Burtschell. 2019. Dosage Effect of Superplasticizer on Selfcompacting Concrete: Correlation Between Rheology and Strength. Journal of Materials Research and Technology, 2019;8(2):2063-2069

[7] Paul Nugraha, Antoni, 2004. Teknologi Beton, Dari Material, Pembuatan, Ke Beton Kinerja Tinggi. Andi, Yogyakarta

[8] PT. SIKA.2016. Product Data Sheet. Sikament NN.

[9] Rahmat, Irma Hendriyani, Moh. Syaiful Anwar, 2016. Analisis Kuat Tekan Beton Dengan Bahan Tambah Reduced Water dan Accelerated Admixture. Info Teknik, Volume 17 No. 2 Desember 2016 (205-218)

[10] Shanti Wahyuni Megasari, Winayati, 2017. Analisis Pengaruh Penambahan Sikament-NN Terhadap Karakteristik Beton. Jurnal Teknik Sipil Siklus, Vol.3, No.2, Oktober 2017

[11] Syafruddin, Hastoro, 2005. Pengaruh Pengurangan Kandungan Air dan Penambahan Superplasticizer pada Komposisi Campuran Beton Kuat Tekan 30 dan 40 Mpa. Tugas Akhir FTSP UII, Yogyakarta

[12] Venu Malagavelli, Neelakanteswara Rao Paturu, 2012. Strength and Workability Characteristics of Concrete by Using Different Super Plasticizers. International Journal of Materials Engineering 2020, 2(1): 7 -11. DOI: 10.5923/j.ijme.20120201.01

[13] Yudi Risdiyanto, 2013. Kajian Kuat Tekan Beton Dengan Perbandingan Volume dan Perbandingan Berat untuk Produksi Beton Massa Menggunakan Agregat Kasar Batu Pecah Merapi (Studi Kasus pada Proyek Pembangunan Sabo Dam). Tugas Akhir Prodi Sipil Universitas Negeri Yogyakarta

[14] Wulfam L. Ervianto, 2006. Eksplorasi Teknologi Dalam Proyek Konstruksi, Beton Pracetak dan Bekisting Edisi I, Andi, Yogyakarta 